\title{
Correspondence
}

\section{Finite-Horizon Prediction of Energy Depletions in Off-Grid Wireless Networks}

\author{
Antti Anttonen, Senior Member, IEEE, \\ Adrian Kotelba, Member, IEEE, \\ Marian Verhelst, Senior Member, IEEE, and \\ Aarne Mämmelä, Senior Member, IEEE
}

\begin{abstract}
In this paper, we present a simple analytic method that can be used to predict potential energy depletion in off-grid wireless backbone network nodes serving mobile users. The instantaneous energy depletion of the batteries of the network nodes is determined by random energy arrivals and departures and modeled as a G/G/1 queue. To evaluate the online energy depletion probability (EDP), an integral-free asymptotic approach is typically used by assuming that the prediction horizon approaches infinity. However, in many practical cases, the time required by user connections can be rather short. This indicates the need for proactive resource management decisions over finite horizons so that the transmission opportunities with limited energy and time horizons are not wasted. Using Hölder's inequality, we obtain a novel finite-horizon upper bound for the EDP, and the result is compared with the infinite-horizon method. The accuracy of the proposed bound, which is addressed both analytically and numerically, proves to be better for shorter prediction horizons. The finiteand infinite-horizon methods are then applied for an energy provisioning admission control (EP-AC) framework using off-grid backbone network nodes. The key observation of this paper is that the proposed finite-horizon prediction approach admits significantly more users to the network when the connection times are relatively short, while retaining an integral-free closed-form structure suitable for the online evaluation of the EDP.
\end{abstract}

Index Terms-Energy depletion, energy harvesting, queueing theory, renewable energy, resource management.

\section{INTRODUCTION}

Resource management methods, such as cooperative routing and connection admission control, have a central role in improving the reliability and energy efficiency of wireless communication networks, [1], [2]. Smart proactive resource management methods become of crucial importance in a rather new class of green wireless networks, where selected on-grid backbone nodes are replaced with off-grid network nodes, which harness local renewable energy to charge their batteries [3]. The interest of the mobile network industry in using renewable energy has recently increased particularly for dense small cells such as femtocells and local area network infrastructure. Under these scenarios, the main expected benefits include easier and faster small-cell network deployments without the need to install on-grid energy and data wires, as well as reduced operational on-grid energy costs and carbon emissions [3].

Manuscript received September 24, 2014; revised January 15, 2015 and May 20, 2015; accepted July 18, 2015. Date of publication August 20, 2015; date of current version August 11, 2016. The review of this paper was coordinated by Prof. F. R. Yu.

A. Anttonen, A. Kotelba, and A. Mämmelä are with VTT Technical Research Centre of Finland, FI-90571 Oulu, Finland (e-mail: antti.anttonen@vtt.fi; adrian.kotelba@vtt.fi; aarne.mammela@vtt.fi).

M. Verhelst is with the Katholieke Universiteit Leuven, 3000 Leuven, Belgium (e-mail: marian.verhelst@esat.kuleuven.be).

Color versions of one or more of the figures in this paper are available online at http://ieeexplore.ieee.org.

Digital Object Identifier 10.1109/TVT.2015.2470087
The dynamic characteristics of the local renewable energy supply and energy demand, however, introduce new challenges in the network design. In essence, energy sustainability, i.e., the prevention of dropping users due to instantaneous energy depletions of the involved energy harvesting nodes, is emphasized. This is a distinctive feature, in comparison with on-grid network nodes, where energy availability is not limited. The energy sustainability problem has been studied for different energy harvesting wireless applications with small cell sizes, including sensor networks [4], [5], local area networks [6], and cellular networks [7], [8]. In these works, it is shown that the provisioned network resource management methods, which take into account potential energy depletion, typically outperform the methods that ignore them.

Due to various stochastic phenomena in mobile networks, the energy sustainability of the network nodes cannot be fully guaranteed. A more practical approach is based on energy provisioning where the energy sustainability is predicted with a given energy depletion probability (EDP). Diffusion approximation, which is based on some past fundamental work [9], [10], has recently been applied to analyze the transient effects of various stochastic energy queue models in network nodes [6]-[8]. Our contribution extends on the work of [6] and the application of the diffusion approximation approach to G/G/1 energy queues for enabling fine-grained energy provisioning admission control (EP-AC).

In [6], the effect of the prediction horizon on the EDP is incorporated through the integration of the probability density function of the first-passage time in which the energy buffer becomes empty. This integral form of the EDP cannot be adopted easily in practice for online prediction in adaptive resource management tasks. The asymptotic upper bound presented in [6] is the first attempt to overcome this drawback in energy-sustainable resource management. Nevertheless, this bound relies on infinite prediction horizon and does not give any insight on how a finite prediction horizon affects the EDP. However, many popular mobile-user data services, such as short messages, e-mails, and short video clips, require finite and relatively short times for which the users are expected to be using the connection [11]. This indicates the need for proactive resource management decisions over finite horizons so that the transmission opportunities with limited energy and time horizons are not wasted. To the best of our knowledge, the advantage of using finite prediction horizons rather than an infinite horizon with G/G/1 energy queue models in the EP-AC concept has yet to be quantified, which has inspired the work for this paper.

In this paper, we first obtain a simple upper bound for the EDP that incorporates the effect of finite prediction horizons in the G/G/1 energy queue model. Similar to [12], in this paper, we aim at trading the accuracy of an integral-based approach for lower evaluation complexity. The accuracy of the bound is addressed both analytically and numerically. The finite-horizon method is then applied for the EP-AC framework using off-grid backbone network nodes. In such energy-limited systems, it is important to minimize the connection blocking probability (CBP) for a given energy depletion risk. The key observation of this paper is that the proposed finite-horizon prediction method, when compared with the infinite-horizon method of [6], admits significantly more users to the network when the connection times are relatively short, while retaining an integral-free structure suitable for online evaluation. The considered EP-AC approach can be readily combined with the resource management that takes into 


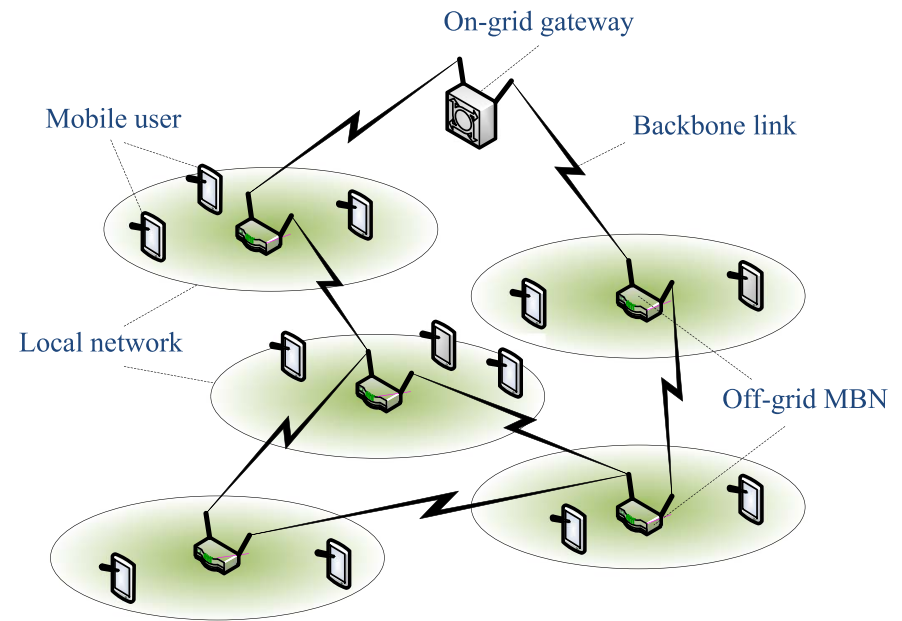

Fig. 1. Wireless mesh backbone network with off-grid MBNs.

account other network restrictions, such as limited bandwidths. Due to space limitations, these aspects are not studied in detail in this paper; see [1].

The remainder of this paper is organized as follows. The target system model is presented in Section II. The EDP prediction and resource management methods are provided in Section III, followed by illustrative performance results in Section IV. Finally, our conclusions are given in Section V.

\section{SYSTEM MODEL}

\section{A. Overview of the EP-AC Concept}

We consider a wireless mesh network topology, which consists of static mesh backbone nodes (MBNs), each serving a number of mobile users (see Fig. 1). In general, MBNs can be access points of a wireless local area network or small base stations of a femtocell network [1], [6], [7]. It is assumed that the MBNs are off-grid nodes charged by a local renewable energy source that can harvest energy provided by sun, wind, etc. To enable a cost-efficient high-density mesh network deployment, only a subset of the nodes acts as an Internet gateway node $(\mathrm{GWN})$ in a given area [1]. The MBNs manage a local network and act as a mesh router that forwards the data requested by mobile users of other MBNs to extend the limited range of the GWN. In the applied EP-AC model, each MBN is characterized by a corresponding node-level EDP, set by the prevailing energy harvesting and energyconsuming traffic conditions over a selected finite time horizon. The GWN then admits a new user to the network only if the EDPs of the MBNs of the selected relay route are below a set target threshold level, which is used to compromise between user blockings and droppings due to energy depletions. The route selection and admission criteria will be detailed in Section III-D.

\section{B. Stochastic Energy Model for MBNs}

The EP-AC approach is affected by the stochastic energy model of the involved MBNs. The dynamic energy buffer of each MBN is represented as an energy queueing system with discrete arrival and departure times of a single energy charge unit over time interval $\left(t_{0}, t_{0}+t\right)$, where $t_{0}$ is the present time, and $t_{0}+t$ denotes the future time instant. Regarding the energy buffer state of the $i$ th MBN, let $b_{i}=q_{i}\left(t_{0}\right)$ denote the initial number of energy units at time $t_{0}, x_{i}(t)$ denote the cumulative number of energy unit arrivals over time interval $\left(t_{0}, t_{0}+\right.$ $t$ ), and $y_{i}(t)$ denote the cumulative number of energy unit departures over time interval $\left(t_{0}, t_{0}+t\right)$ with $b_{i}>0$ and $x_{i}\left(t_{0}\right)=y_{i}\left(t_{0}\right)=0$. Then, the number of energy units in the energy buffer of the $i$ th MBN at time $t_{0}+t$ can be represented as follows (cf. [6] and [8]):

$$
q_{i}\left(t_{0}+t\right)=\min \left\{\max \left\{b_{i}+x_{i}\left(t_{0}+t\right)-y_{i}\left(t_{0}+t\right), 0\right\}, B_{i}\right\}
$$

where $B_{i} \geq b_{i}$ is the maximum energy level of the $i$ th energy buffer. We assume that $0 \leq t \leq S$, where $S>0$ denotes the selected finite prediction horizon in time. Following the normalized model of [6], [8], the energy charge level is represented by the number of energy units where one energy unit can take any value in joules. Correspondingly, the prediction horizon time $S$ is represented in terms of a number of time units, where one time unit can take any value in seconds. For analytic tractability, in the theoretical analysis, we assume that the potential energy buffer overflows do not affect the EDP. As will be demonstrated in Section IV, this is a reasonable assumption for static MBNs equipped with energy buffers having a relatively large capacity. An energy depletion is declared at the $i$ th node if $q_{i}(t)$ becomes zero.

In general, there are a number of alternative system-specific sources that affect the stochastic behavior of energy arrivals and departures [1], [3], [6]-[8]. Modeling of the energy-dependent components individually for a specific system is beyond the scope of this paper. Without loss of generality, we apply a customary G/G/1 queue model approach from [6]-[8]. In this framework, the involved distributions of the energy interarrival and interdeparture times, associated with $x_{i}(t)$ and $y_{i}(t)$, are unknown to the MBNs, except their respective means $m_{x_{i}}$ and $m_{y_{i}}$, and standard deviations $\sigma_{x_{i}}$ and $\sigma_{y_{i}}$. For analytic tractability, we adopt a customary assumption that the interarrival and interdeparture times are independent, as was done in [6]-[8]. The extension to a nonstationary system with slowly time-varying moments and online moment estimation is possible because a nonstationary $\mathrm{G} / \mathrm{G} / 1$ system can be decomposed into a series of stationary G/G/1 systems, as shown in [10].

\section{Proposed Energy Depletion Prediction Method}

Here, after providing the relevant theoretical background, we present a novel expression for the EDP that incorporates the finite prediction horizons in the G/G/1 energy buffer model. The accuracy of the proposed method is then addressed analytically, and finally, the method is applied for the EP-AC framework.

\section{A. Theoretical Background}

We apply the well-known diffusion approximation approach used, e.g., in [6]-[10], as the basis for the analysis. We shortly review the main assumptions leading to the diffusion approximation model (for a more detailed treatment, see [6], [9], and [10] and the references therein). The distributions of the energy interarrival and interdeparture times can be general and do not need to be specified. Yet, the model does require the knowledge of their first- and second-order statistical moments. We drop the node index $i$ because the calculation of the EDP is essentially the same for all the MBNs. The random energy level $q(t)$ is modeled as the continuous-time Brownian motion with an absorbing barrier at $q(t)=0$. Specifically, the density of the incremental change of energy buffer state $\Delta q(t)=q(t+\Delta)-q(t)$ between time $t$ and time $t+\Delta$ is assumed to converge to the normal distribution as $\Delta q(t) \approx \beta \Delta+z(t) \sqrt{\alpha \Delta}$, where $z(t)$ is the standard white Gaussian process, $\beta=m_{x}^{-1}-m_{y}^{-1}$ is the drift coefficient, and $\alpha=\sigma_{x}^{2} m_{x}^{-3}+\sigma_{y}^{2} m_{y}^{-3}$ is called the diffusion coefficient [6]. Obviously, the more independent events that occur during the observation time window, the better the convergence will be. This is justified by the central limit theorem, which introduces the requirement that the interarrival and interdeparture times are independent and identically distributed random variables. 
Without loss of generality, let $t_{0}=0, \alpha>0$, and $\beta \in \mathbb{R}$, where $\mathbb{R}$ denotes the set of real numbers. Using the given diffusion approach, the EDP is defined as $\mathcal{P}=\operatorname{Pr}\{\phi(t)<S\}$, where $\phi(t)=\inf \{t$ : $q(t)=0 \mid q(0)=b\}$ denotes the first-passage time from $q(0)=b$ to $q(t)=0, \operatorname{Pr}\{\cdot\}$ denotes the probability, and $\inf \{\cdot\}$ is the infimum function. The EDP is obtained from the following integration [6]:

$$
\mathcal{P}=\int_{0}^{S} \frac{b}{\sqrt{2 \pi \alpha t^{3}}} \exp \left[-\frac{(\beta t+b)^{2}}{2 \alpha t}\right] d t .
$$

For a sufficiently large value of $S$, (2) can be approximated by [6]

$$
\mathcal{P} \leq \lim _{S \rightarrow \infty} \mathcal{P}=\exp \left(-\frac{b|\beta|+b \beta}{\alpha}\right)
$$

\section{B. New Upper Bound for the EDP}

The upper bound (3) is interesting because it is simple enough to be applied for practical systems, which require online performance prediction capability. However, (3) fails to approximate the EDP accurately unless $S$ is sufficiently large. Next, we will derive a new upper bound to (2) that can incorporate $S$, while retaining a lowcomplexity integral-free expression.

Theorem 1: If $\beta \neq 0$, then for a given prediction horizon $S$, the EDP (2) is upper bounded by the following:

$$
\begin{aligned}
\mathcal{P} \leq \frac{b}{\sqrt{2 \pi \alpha}} & \exp \left(-\frac{2 \beta b S+b^{2}}{2 \alpha S}\right)\left(\sum_{k=0}^{n} \frac{n !}{k !} \frac{S^{-k}}{\mu^{n-k+1}}\right)^{\frac{1}{p}} \\
& \times\left\{\frac{2 \alpha(p-1)}{p \beta^{2}}\left[1-\exp \left(-\frac{p \beta^{2} S}{2 \alpha(p-1)}\right)\right]\right\}^{\frac{p-1}{p}}
\end{aligned}
$$

where $n=0,1,2, \ldots, p=(2 n+4) / 3$, and $\mu=p b^{2} /(2 \alpha)$.

Proof: We first observe that integral (2) can be rewritten as $\mathcal{P}=w \int_{0}^{S} f(t) g(t) d t$, where $f(t)=t^{-3 / 2} \exp \left(-b^{2} /(2 \alpha t)\right), g(t)=$ $\exp \left(-\beta^{2} t /(2 \alpha)\right)$, and $w=(b / \sqrt{2 \pi \alpha}) \exp (-\beta b / \alpha)$. This form enables the use of Hölder's inequality [13, Eq. 12.312, pp. 1131] as $\mathcal{P} \leq w\left(\int_{0}^{S}|f(t)|^{p} d t\right)^{1 / p}\left(\int_{0}^{S}|g(t)|^{p /(p-1)} d t\right)^{(p-1) / p}$, where $p>$ 1 is the parameter used to manipulate the tightness of Hölder's inequality. After the change of variables $z=t^{-1}$ and $\mu=p b^{2} /(2 \alpha)$, and using [13, Eq. 3.351.2, pp. 357], we obtain $\int_{0}^{S}|f(t)|^{p} d t=$ $\exp \left(-p b^{2} /(2 \alpha S)\right) \sum_{k=0}^{n}(n ! / k !)\left(S^{-k} / \mu^{n-k+1}\right)$ where $n=(3 p-$ $4) / 2$ is necessarily a nonnegative integer. If $\beta \neq 0$, then using [13, Eq. 3.351.1, pp. 357], we obtain $\int_{0}^{S}|g(t)|^{p /(p-1)} d t=2 \alpha(p-$ $1) /\left(p \beta^{2}\right)\left[1-\exp \left(-p \beta^{2} S /(2 \alpha(p-1))\right)\right]$. Finally, (4) is obtained by substituting the given expressions into Hölder's inequality.

Corollary 1: If $\beta=0$, then for a given prediction horizon $S$, the EDP in (2) is upper bounded by the following:

$$
\mathcal{P} \leq \frac{b S^{\frac{p-1}{p}}}{\sqrt{2 \pi \alpha}} \exp \left(-\frac{b^{2}}{2 \alpha S}\right)\left(\sum_{k=0}^{n} \frac{n !}{k !} \frac{S^{-k}}{\mu^{n-k+1}}\right)^{\frac{1}{p}} .
$$

Proof: If $\beta=0$, then $\int_{0}^{S}|g(t)|^{p /(p-1)} d t=S$, which leads to (5) after rearrangement of the terms.

Corollary 2: If $p=2$, then for a given prediction horizon $S$, (4) and (5) reduce to

$$
\mathcal{P} \leq \omega(\beta) \exp \left(-\frac{2 \beta b S+b^{2}}{2 \alpha S}\right)
$$

where $\omega(\beta)=\sqrt{\alpha\left(b^{2}+\alpha S\right) /\left(2 \pi b^{2} \beta^{2} S\right)\left[1-\exp \left(-\beta^{2} S / \alpha\right)\right]}$ for $\beta \neq 0$, and $\omega(0)=\sqrt{\left(b^{2}+\alpha S\right) /\left(2 \pi b^{2}\right)}$ for $\beta=0$.
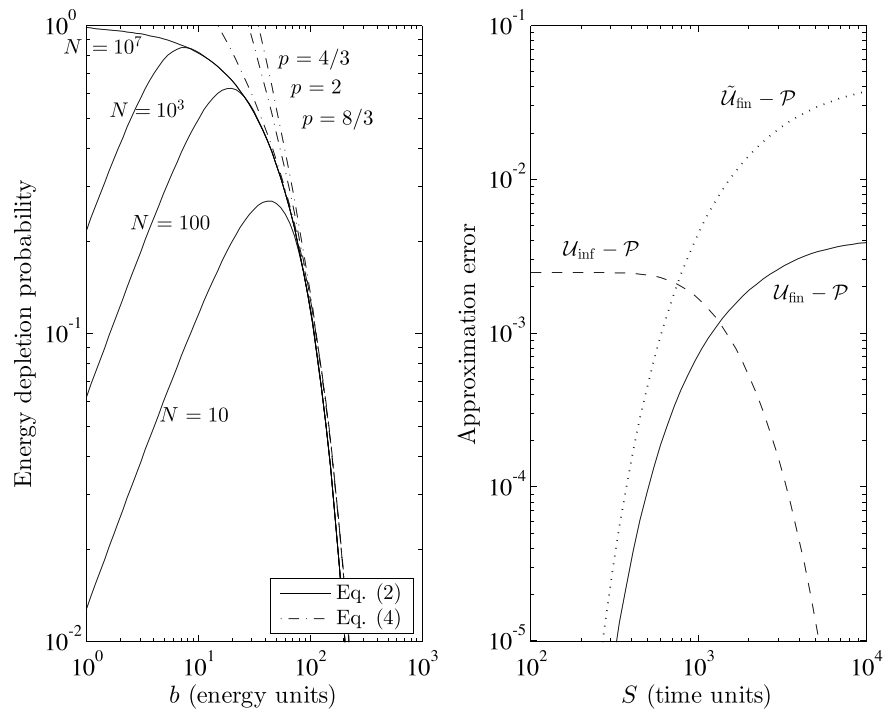

Fig. 2. Numerical illustration of the approximation characteristics for different EDP evaluation methods.

Proof: The proof is straightforward by using (4) and (5) and rearrangement of the terms.

The EDP from (2) and its upper bound (4) are shown in the left-hand side of Fig. 2. For comparison purposes, the integral (2) is evaluated using $N$-point extended trapezoidal numerical integration method [14], where $N$ denotes the required number of integrand evaluations in (2) with different time intervals. The results are shown for $\beta=0.2$, $\alpha=20, S=1000$, and the three lowest values of $p$. In essence, it is difficult to ensure that a low value of $N$, enabling a low-complexity solution, is not seriously underestimating the EDP due to the irregularity of the integrand in (2). We remark that underestimation of the EDP is much more harmful for the EP-AC concept than overestimation of the EDP because the necessary condition for the connection admission is to ensure that the EDP is below a target threshold. Obviously, any underestimation of the EDP from (2) is avoided by using the upper bound (6). Furthermore, we observe from (6) and the left-hand side of Fig. 2 that the calculation of relatively complicated high-order roots in (4) can be avoided by selecting the value $p=2$ without significantly affecting the accuracy. Therefore, we next focus on the approximation error of (6), which is applied for the EP-AC framework in Sections III-D and IV.

\section{Analytic Assessment of Approximation Error Characteristics}

Let $\mathcal{U}_{\text {inf }} \geq \mathcal{P}$ denote the infinite-horizon bound obtained from (3) and $\mathcal{U}_{\text {fin }} \geq \mathcal{P}$ denote the finite-horizon bound obtained from (6). Consequently, the corresponding nonnegative approximation errors are $\varepsilon_{\text {inf }}=\mathcal{U}_{\text {inf }}-\mathcal{P}$ and $\varepsilon_{\text {fin }}=\mathcal{U}_{\text {fin }}-\mathcal{P}$. It is easy to show that $\varepsilon_{\text {inf }} \rightarrow 0$, for all $\beta, \alpha, b$, when $S$ approaches infinity and $\varepsilon_{\text {inf }} \rightarrow$ $\exp (-(b|\beta|+b \beta) / \alpha)$ when $S$ approaches zero.

Theorem 2: The approximation error of the finite-horizon bound is as follows:

$$
\varepsilon_{\text {fin }} \leq \mathcal{U}_{\text {fin }}-\sqrt{\max \left\{0, \mathcal{U}_{\text {fin }}^{2}-\frac{b^{2} M_{1}^{2}}{6 \pi \alpha S} \exp \left(-\frac{2 b \beta}{\alpha}\right)\right\}}
$$

where $M_{1}=\exp \left(-b^{2} /(2 \alpha S)\right)$ for $S \leq b^{2} /(3 \alpha)$, and $M_{1}=$ $\sqrt{27 S^{3} \alpha^{3} / b^{6}} \exp (-3 / 2)$ for $S>b^{2} /(3 \alpha)$.

Proof: Rewrite integral (2) as $\mathcal{P}=w / \sqrt{S} \int_{0}^{1} f(z) g(z) d z$, where $f(z)=z^{-3 / 2} \exp \left(-b^{2} /(2 \alpha S z)\right)$, and $g(z)=\exp \left(-\beta^{2} S z /(2 \alpha)\right)$. Using Ozeki's inequality $[15$, Eq. 4.2] and the identity 
1: if an admission request arrives to one of the MBNs then

for $j=1$ to $J$ do

$\breve{\mathcal{P}}_{j} \leftarrow 0$

for $i=1$ to $L_{j}$ do

Obtain $\mathcal{P}_{i, j}$ for given $\left(\beta_{i, j}, \alpha_{i, j}, b_{i, j}, S\right)$

$\breve{\mathcal{P}}_{j} \leftarrow \max \left\{\mathcal{P}_{i, j}, \breve{\mathcal{P}}_{j}\right\}$

Send $\breve{\mathcal{P}}_{j}$ to the next MBN until GWN is reached end for

end for

GWN determines $j_{\text {min }} \in(1, J)$ that has the lowest $\breve{\mathcal{P}}_{j}$

if $\breve{\mathcal{P}}_{j_{\min }} \leq \mathcal{P}_{0}$ then

GWN admits the new user to the network

GWN selects route $j_{\min }$ for the data transfer

else

GWN blocks the new connection request end if

end if $\triangleright$ Activation of admission control

$\triangleright$ Loop for the jth candidate route

$\triangle$ Loop for the ith MBN of the jth route

$\triangleright$ Finite-horizon depletion prediction

$\triangleright$ GWN needs only one EDP per route

$\triangleright$ Route selection criterion $\triangleright$ Admission decision criterion

Fig. 3. Pseudocode for the EP-AC procedure in a mesh backbone network using off-grid nodes.

$\mathcal{U}_{\text {fin }}^{2}-\mathcal{P}^{2}=-\varepsilon_{\text {fin }}^{2}+2 \mathcal{U}_{\text {fin }} \varepsilon_{\text {fin }}$, we obtain the quadratic expression $-\varepsilon_{\text {fin }}^{2}+2 \mathcal{U}_{\text {fin }} \varepsilon_{\text {fin }}-w^{2} /(3 S)\left(M_{1} M_{2}-m_{1} m_{2}\right)^{2} \leq 0$, where $0 \leq m_{1} \leq f(z) \leq M_{1}$, and $0 \leq m_{2} \leq g(z) \leq M_{2}$. The function $f(z)$ is monotonically increasing for $z<z^{*}$ and monotonically decreasing for $z>z^{*}$ where $z^{*}=b^{2} /(3 S \alpha)$ is the point in which $f(z)$ achieves its global maximum. Using l'Hôpital's rule, we obtain $m_{1}=$ $\lim _{z \rightarrow 0} f(z)=0$, regardless of the value of $S$. On the other hand, if $S>b^{2} /(3 \alpha)$, then $z^{*}<1$ and $M_{1}=f\left(z^{*}\right)$. Otherwise, if $S \leq b^{2} /(3 \alpha)$, then $z^{*} \geq 1$ and $M_{1}=f(1)$. It can readily be shown that $m_{2}=\exp \left(-\beta^{2} S /(2 \alpha)\right)$ and $M_{2}=1$, which finally lead to (7) after solving the above quadratic error equation for the real solutions.

Corollary 3: The approximation error $\varepsilon_{\mathrm{fin}} \rightarrow 0$ for all $\beta, \alpha, b$, when $S$ approaches zero.

Proof: Using (6) and (7), we first obtain $\lim _{S \rightarrow 0^{+}} \mathcal{U}_{\text {fin }}=$ $(1 / \sqrt{2 \pi}) \exp (-\beta b / \alpha) \lim _{S \rightarrow 0^{+}} \exp \left(-b^{2} /(2 \alpha S)\right)=0$. Furthermore, since $\lim _{S \rightarrow 0^{+}} b^{2} M_{1}^{2} /(\alpha S)=\lim _{S \rightarrow 0^{+}} \exp \left(-b^{2} /(\alpha S)\right)=0$, we immediately obtain $\lim _{S \rightarrow 0}+\varepsilon_{\text {fin }}=0$ after substituting the two limits into (7).

Based on the above analytic characteristics of $\varepsilon_{\text {inf }}$ and $\varepsilon_{\text {fin }}, \mathcal{U}_{\text {fin }}$ clearly complements $\mathcal{U}_{\text {inf }}$ by improving the accuracy for shorter prediction horizons. Since both $\mathcal{U}_{\text {fin }}$ and $\mathcal{U}_{\text {inf }}$ are upper bounds of $\mathcal{P}$, the smaller bound gives a smaller approximation error. This immediately leads to a composite bound $\mathcal{P} \leq \min \left\{\mathcal{U}_{\text {fin }}, \mathcal{U}_{\text {inf }}\right\}$ whose error $\min \left\{\varepsilon_{\text {fin }}, \varepsilon_{\text {inf }}\right\} \rightarrow 0$ when $S$ approaches either to zero or infinity. We remark that the complexity of $\mathcal{U}_{\text {fin }}$ could be further reduced by setting $\omega(\beta)=1 \forall \beta, \alpha, b, S$ at the cost of affecting the accuracy that is proportional to $1-\omega(\beta)$ with $\omega(\beta)<0$. As a result, $\mathcal{U}_{\text {fin }}$ would reduce to $\tilde{\mathcal{U}}_{\text {fin }}=\exp \left(-\left(2 \beta b S+b^{2}\right) /(2 \alpha S)\right)$, which can also be deduced in the context of evaluating video interruption probability [11]. The given observations of the characteristics of the approximation errors are numerically illustrated with $\beta=0.2, \alpha=20$, and $b=300$ on the right-hand side of Fig. 2.

\section{Application to the EP-AC Framework}

We now apply the proposed upper bound (6) to the EP-AC scenario described in Section II. Consider a $j$ th $(j \in(1, J))$ possible route between the destination MBN, from which a new randomly arriving user is requesting a data service, and the closest GWN via $L_{j}$ hops over relay MBNs. The random state of the network, which determines the admission decision, is solely defined by the predicted EDPs $\mathcal{P}_{i, j}$ for the given set $\left(S, b_{i, j}, \beta_{i, j}, \alpha_{i, j}\right)$. The prediction horizon $S$ is defined as the connection time that the users expect to be using the data services via the MBNs. The subindex $(\cdot)_{i, j}$ denotes the $i$ th MBN in the $j$ th candidate route, where the subindex $j$ is dropped if not necessary for the context. The EP-AC method first selects a route $j_{\text {min }}$ with the lowest $\breve{\mathcal{P}}_{j}$, where $\breve{\mathcal{P}}_{j}=\max _{i}\left(\mathcal{P}_{i, j}\right)$. To avoid connection droppings due to potential energy depletion of the involved MBNs during a finite $S$, the admission is granted by the GWN if $\breve{\mathcal{P}}_{j_{\text {min }}}$ is below a given threshold $\mathcal{P}_{0}$, i.e., if $\mathcal{P}_{i, j_{\min }} \leq \mathcal{P}_{0} \forall i \in\left(1, L_{j_{\min }}\right)$. Otherwise, the connection request is blocked, affecting the CBP that is defined as $\operatorname{Pr}\left(\breve{\mathcal{P}}_{j_{\text {min }}}>\mathcal{P}_{0}\right)$. The estimation of the MBN-dependent means and standard deviations of energy arrivals and departures, required to calculate $\mathcal{P}_{i, j}$, can be performed via the standard sample moment estimation methods [16]. The detailed design of the required handshaking and control signaling, which does not affect the results of Section IV, is not presented due to space limitations. The resulting EP-AC procedure is presented in Fig. 3.

\section{Performance Results}

Here, the EP-AC protocol from Section III is applied for deciding whether a new arriving user can be admitted without violating the EDP-based admission decision rule calculated either via the finitehorizon or infinite-horizon method. The main goal is to demonstrate the benefits from inclusion of the finite time horizons to the EP-AC concept in a selected mesh backbone network scenario.

\section{A. Simulation Setup and Used Energy Profiles for MBNs}

Following [6], the EP-AC model is captured by the stochastic energy harvesting and consumption processes of the MBNs, using the G/G/1 model from Section II. We evaluate a mesh backbone network with one GWN and five partially connected MBNs, as shown in Fig. 1. For testing purposes, we assume that the energy interarrival times associated with $x_{i}(t)$ follow a discrete probability mass function (pmf), as was done in [6]. Specifically, we assume a two-state energy harvesting source representing a high-energy arrival state (e.g., direct sunlight or turbulent wind) and a low-energy arrival state (e.g., indirect sunlight or light wind), selected with the given pmf. To harvest a 
single energy unit in the $i$ th MBN, the low and high harvesting states are assumed to require one time unit with probability of $v_{i}$ and a fraction of the time unit equivalent to 0.1 with probability of $1-v_{i}$, respectively. Following [6], the energy consumption process $y_{i}(t)$ is characterized by converting the random traffic arrivals into random energy consumption events. Each random traffic event is set to consume one energy unit. We assume that the interdeparture times of energy units follow the Weibull distribution, which is associated with the scale $\theta_{i}$ and shape $\eta_{i}$ parameters, representing the prevailing heterogeneous energy demands of each MBN. The relationship between $\left(v_{i}, \theta_{i}, \eta_{i}\right)$ and $\left(m_{x_{i}}, \sigma_{x_{i}}, m_{y_{i}}, \sigma_{y_{i}}\right)$ to determine $\left(\beta_{i}, \alpha_{i}\right)$ is not presented due to space limitations, but the result can readily be found using the known characteristics of the pmf and Weibull distributions [16].

In the following examples, we examine the effect of the known statistical parameters of random energy arrival and consumption processes for given finite connection times $S$ and initial energy levels $b_{i}$. Our objective is twofold.

First, in Section IV-B, the characteristics of different EDP evaluation methods are compared in the defined provisioned admission control task. We examine the EDP of two different node types individually, and their statistical parameters are fixed as follows. For the node $i=1$, we set $v_{1}=0.6, \theta_{1}=0.73$, and $\eta_{1}=2.00$, leading to $\beta_{1}=0.018$ and $\alpha_{1}=1.16$. For the node $i=2$, we set $v_{2}=0.3$, $\theta_{2}=0.36$, and $\eta_{2}=1.00$, leading to $\beta_{2}=-0.10$ and $\alpha_{2}=6.16$.

Second, in Section IV-C, we apply the proposed EDP method to the EP-AC by including the whole network with six nodes and simulating the resulting CBP, i.e., $\operatorname{Pr}\left(\breve{\mathcal{P}}_{j_{\text {min }}}>\mathcal{P}_{0}\right)$, which is analytically unknown for randomly changing statistical parameters. The purpose is to demonstrate how the proposed finite-horizon prediction method, when compared with the infinite-horizon one, can reduce the $\mathrm{CBP}$ of the EP-AC approach while keeping the target $\mathcal{P}_{0}$. Our emphasis is on demonstrating the effect of different proportions of asymptotically underresourced $\left(\beta_{i}<0\right)$ and overresourced $\left(\beta_{i}>0\right)$ MBNs. To obtain a desired range of $\beta_{i}$ in the network, the statistical parameters $\left(v_{i}, \theta_{i}, \eta_{i}\right)$ of each MBN and admission trial are randomly selected from the uniform distribution with the given limits. The condition $-m_{y_{i}}^{-1} \leq \beta_{i} \leq m_{x_{i}}^{-1}$ ensures that $m_{x_{i}}$ and $m_{y_{i}}$ are nonnegative. The simulation results are obtained using $10^{5}$ experiments.

\section{B. EDP Evaluation Comparison to Provision Admission Control}

The finite- and infinite-horizon EDP evaluation methods are used in Fig. 4 for the two MBNs with $i \in(1,2), b_{1}=100$, and $b_{2}=80$, and different energy profiles, as defined in Section IV-A. Unlike the infinite-horizon bound, the proposed bound (6) is able to follow (2) quite closely. We notice that the bound (6) is not uniformly better than the infinite-horizon bound, slightly degrading the asymptotic convergence in comparison with the infinite-horizon method (3). Further improvements may hence be obtained by using a composite approach in which (3) and (6) are used jointly, as discussed in Section III-C.

In Fig. 5, the purpose is to illustrate how the assumptions on infinite energy buffer size and infinite prediction horizon affect the relative accuracy of evaluating the EDP with $S=100$ and $S=1000$ for the nodes $i=1$ and $i=2$, respectively. The approximation error, resulting from assuming infinite buffer size, is the largest when the buffer is full. However, the error diminishes as the energy buffer size gets larger. This suggests that, for large energy buffer sizes, the error resulting from assuming infinite buffer size is relatively small. Therefore, since it is difficult to encounter both effects analytically, it is more important to include the effect of a finite prediction horizon rather than a finite buffer size.

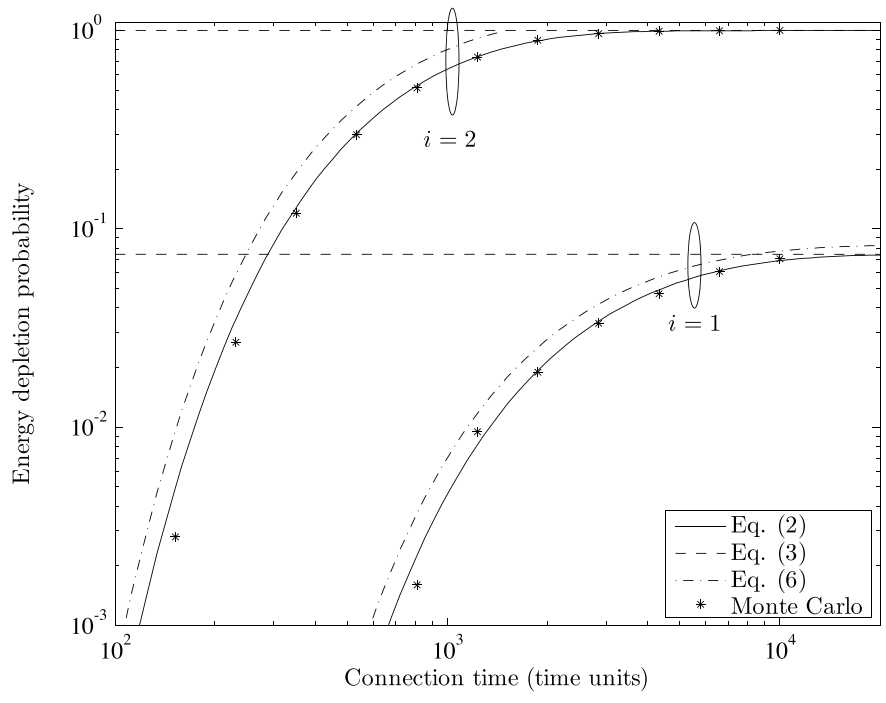

Fig. 4. Comparison of the EDP evaluation methods to predict energy depletion with two different MBNs for a given connection time $S$ and infinite energy buffer size.

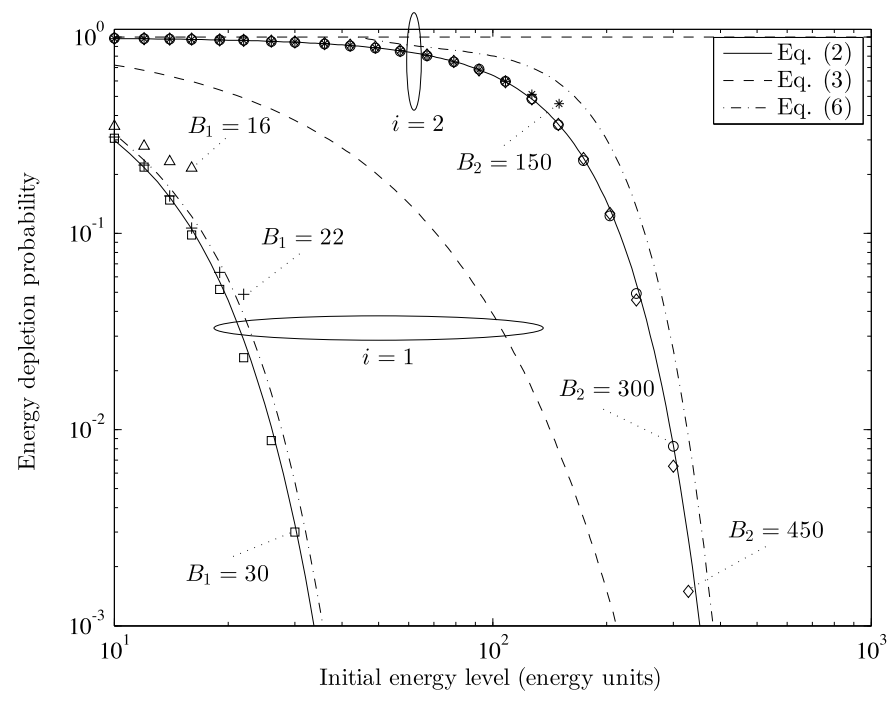

Fig. 5. Effect of the finite energy buffer size $B_{i}$ on EDP evaluation accuracy with two different MBNs for a given initial energy level $b_{i} \leq B_{i}$. The markers represent the results using the Monte Carlo method for different finite energy buffer sizes.

\section{CBP of Finite- and Infinite-Horizon EP-AC}

Next, we illustrate the CBP when the EP-AC is based on a known connection time $S$ (finite horizon) and when the finite connection time is not used (infinite horizon). In the former case, the EDP is calculated using (6), whereas in the latter case, the EDP is calculated with (3). In Figs. 6 and 7, the CBP is plotted as a function of $S$ and $b_{i}$ using $\mathcal{P}_{0}=0.05$. It is seen that the shorter the connection time or larger the initial energy level, the lower the probability that the energy will be depleted and, therefore, the lower the CBP. When the connection time is relatively limited, the proposed finite-horizon admission control method is more likely to admit users to the network than the infinite-horizon method, while still keeping the probability of an energy depletion below $\mathcal{P}_{0}$ at each involved MBN. Since the infinite-horizon method is incapable of recognizing the transmission opportunities for $\beta_{i} \leq 0$, one observes a significantly higher CBP for the infinite-horizon method when asymptotically underresourced nodes are dominating the network. 


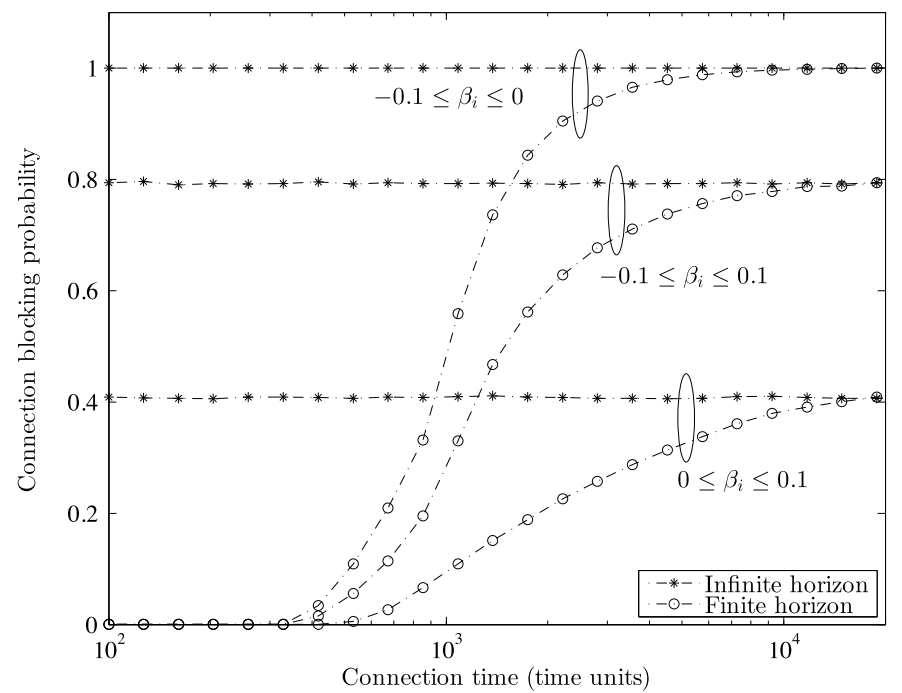

Fig. 6. Simulated CBP with infinite- and finite-horizon EP-AC for a given connection time $S$ with different proportions of asymptotically underresourced and overresourced MBNs $\left(b_{i}=200\right)$.

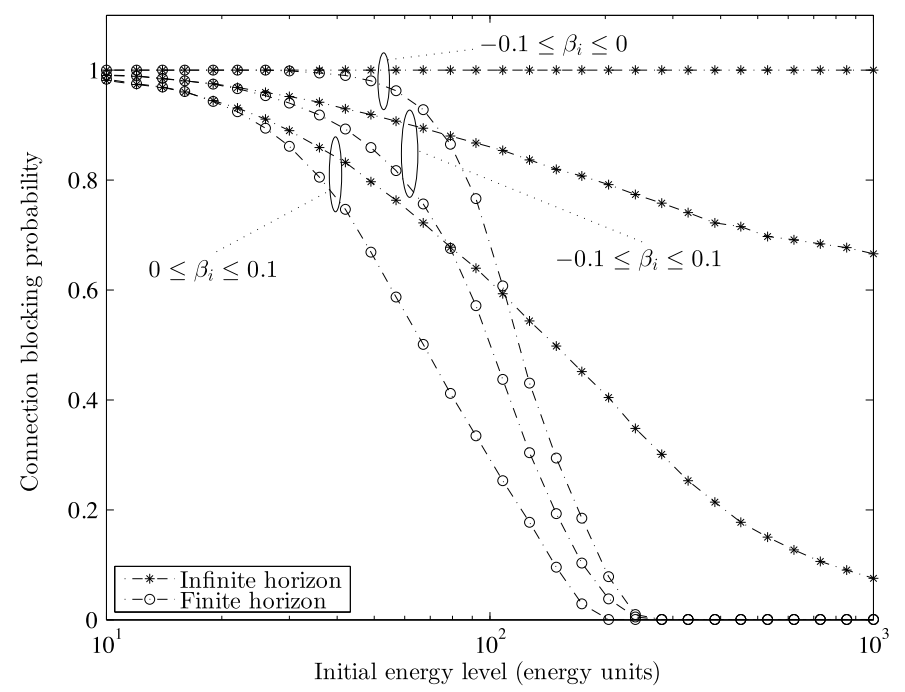

Fig. 7. Simulated CBP with infinite- and finite-horizon EP-AC for a given initial energy level $b_{i}$ with different proportions of asymptotically underresourced and overresourced MBNs $(S=500)$.

\section{CONCLUSION}

In this paper, we have presented a novel low-complexity finitehorizon EDP method and its application to an EP-AC framework. The merits of the proposed finite-horizon EP-AC approach, when compared with the infinite-horizon one, can be seen from its capability to admit significantly more users to join the network when the connection times are relatively short, while retaining an integral-free structure suitable for online evaluation. The reason behind the improvement is that the transmission opportunities with limited energy and time horizons are not wasted, most notably for asymptotically underresourced nodes. The proposed upper bound leaves a residual approximation error that approaches zero as the prediction horizon tends to zero. In case a wide range of prediction horizons must be supported, a combination of the finite- and infinite-horizon methods can be used to further reduce the approximation error. In many practical cases, one is not necessarily interested in knowing the exact EDP but rather in knowing that the maximum EDP is below some target value. We have proven that the proposed approximation method has an upper bound characteristic for all possible parameter values affecting the EDP, making it an adequate choice for practical usage. We expect that other bounds with different tradeoffs between accuracy and computational complexity could still be found. This, as well as the combination of the EP-AC approach with the resource management considering limited bandwidths, can pose interesting opportunities for future work.

\section{ACKNOWLEDGMENT}

The authors would like to thank Prof. S. Pollin, Dr. F. Rosas, and the anonymous reviewers for their critical and constructive comments that helped in significantly improving the quality of this paper.

\section{REFERENCES}

[1] P. Pathak and R. Dutta, "A survey of network design problems and joint design approaches in wireless mesh networks," IEEE Commun. Surveys Tuts., vol. 13, no. 3, pp. 396-428, 3rd Quart. 2011.

[2] M. Ahmed, "Call admission control in wireless networks: A comprehensive survey," IEEE Commun. Surveys Tuts., vol. 7, no. 1, pp. 50-69, 1st Quart. 2005.

[3] G. Piro et al., "HetNets powered by renewable energy," IEEE Internet Comput., vol. 17, no. 1, pp. 32-39, Jan. 2013.

[4] N. Roseveare and B. Natarajan, "An alternative perspective on utility maximization in energy-harvesting wireless sensor networks," IEEE Trans. Veh. Technol., vol. 63, no. 1, pp. 344-356, Jan. 2014.

[5] D. Gunduz, K. Stamatiou, N. Michelusi, and M. Zorzi, "Designing intelligent energy harvesting communication systems," IEEE Commun. Mag., vol. 52, no. 1, pp. 210-216, Jan. 2014.

[6] L. Cai et al., "Sustainability analysis and resource management for wireless mesh networks with renewable energy sources," IEEE J. Sel. Areas Commun., vol. 32, no. 2, pp. 345-355, Feb. 2014.

[7] Y. Xu, H. Li, Z. Feng, P. Zhang, and S. Ci, "Energy sustainability modeling and liquid cell management in green cellular networks," in Proc. IEEE Int. Conf. Commun., Budapest, Hungary, 2013, pp. 4414-4419.

[8] X. Zhang et al., "Optimizing network sustainability and efficiency in green cellular networks," IEEE Trans. Wireless Commun., vol. 13, no. 2, pp. 1129-1139, Feb. 2014

[9] R. Cox and H. Miller, The Theory of Stochastic Processes. London, U.K.: Chapman \& Hall, 1965.

[10] A. Duda, "Diffusion approximation for time-dependent queueing systems," IEEE J. Sel. Areas Commun., vol. JSAC-4, no. 6, pp. 905-918, Sep. 1986.

[11] A. Anttonen and A. Mämmelä, "Interruption probability of wireless video streaming with limited video lengths," IEEE Trans. Multimedia, vol. 16, no. 4, pp. 1176-1180, Jun. 2014.

[12] M. Simon and M.-S. Alouini, "Exponential-type bounds on the generalized Marcum Q-function with application to error probability analysis over fading channels," IEEE Trans. Commun., vol. 48, no. 3, pp. 359-366, Mar. 2000.

[13] I. Gradshteyn and I. Ryzhik, Table of Integrals, Series, and Products. London, U.K.: Academic, 1994.

[14] W. Press, B. Flannery, S. Teukolsky, and W. Vetterling, Numerical Recipes. New York, NY, USA: Cambridge Univ. Press, 2007.

[15] S. Izumino, H. Mori, and Y. Seo, "On Ozeki's inequality," J. Inequalities Appl., vol. 2, no. 3, pp. 235-253, 1998.

[16] E. Lehmann and G. Casella, Theory of Point Estimation. New York, NY, USA: Springer-Verlag, 1998. 